\title{
Influência de materiais recicláveis e vinhaça da cana de açúcar na resistência
}

\section{mecânica de tijolos ecológicos}

\author{
Influence of recyclable materials and sugar cane vinasse on the mechanical strength of ecological \\ bricks
}

Influencia de materiales reciclables y vinaza de caña de azúcar en la resistencia mecánica de

ladrillos ecológicos

Recebido: 12/02/2021 | Revisado: 21/02/2021 | Aceito: 21/02/2021 | Publicado: 28/02/2021

\author{
Maria Eunice Carvalho Tosello \\ ORCID: https://orcid.org/0000-0001-8582-4997 \\ Universidade do Oeste Paulista, Brasil \\ E-mail: arqtosello1@gmail.com \\ Jacqueline Roberta Tamashiro \\ ORCID: https://orcid.org/0000-0001-5607-6555 \\ Universidade do Oeste Paulista, Brasil \\ E-mail: jtamashiro@outlook.com.br \\ Lucas Henrique Pereira Silva \\ ORCID: https://orcid.org/0000-0002-8338-5609 \\ Instituto Federal de São Paulo, Brasil \\ E-mail: lucashpsilva@ifsp.edu.br \\ Patrícia Alexandra Antunes \\ ORCID: https://orcid.org/0000-0003-2173-9712 \\ Universidade do Oeste Paulista, Brasil \\ E-mail: antunes@unoeste.br \\ Rebeca Delatore Simões \\ ORCID: https://orcid.org/0000-0002-4556-3718 \\ Universidade Estadual Paulista, Brasil \\ E-mail: rebeca.simoes@unesp.br
}

\begin{abstract}
Resumo
A indústria da construção civil utiliza abundante matéria-prima não renovável, consome grande quantidade de energia na extração de insumos, na produção dos materiais, no transporte de ambos e gera uma infinidade de entulho na execução das obras. Tijolos ecológicos podem ser fabricados utilizando resíduos urbanos atóxicos, incluindo os da construção civil. Este trabalho objetivou descrever o processo de fabricação e a caracterização estrutural de tijolos ecológicos utilizando como matriz o argissolo e o latossolo, e como aglomerante, materiais recicláveis da coleta seletiva urbana (PET e EPS), reboco descartado pela construção civil e a vinhaça da indústria canavieira. Foram realizados estudos do processo de fabricação de protótipos de tijolos ecológicos e da caracterização mecânica: absorção de água, densidade seca, resistência à compressão mecânica, comportamento tensão/deformação, comportamento massa/resistência à compressão. Os resultados mostraram que a inclusão acima de excessiva de materiais recicláveis na fabricação dos adobes promove o aumento da resistência mecânica, mas também aumenta a absorção de água de forma a não atender a regulamentação da norma brasileira que dispõe sobre o tijolo maciço de solo-cimento. Os tijolos mais promissores foram os fabricados com 55 e 39,5\% de materiais recicláveis para o argissolo e o latossolo respectivamente. Palavras-chave: Adobe; Argissolo; Latossolo; PET; EPS; Compressão mecânica.
\end{abstract}

\begin{abstract}
The construction industry uses abundant non-renewable raw materials, consumes large amounts of energy in the extraction of inputs, in the production of materials, in the transport of both and generates an infinity of rubble in the execution of the works. Ecological adobe bricks can be manufactured using non-toxic urban waste, including civil construction. This work aimed to describe the manufacturing process and the structural characterization of ecological bricks of the adobe type using as a matrix the argisol and the oxisol, and as a binder, recyclable materials from the selective urban collection (PET and EPS), plaster discarded by the civil construction and the vinasse from the sugarcane industry. Studies were carried out on the manufacturing process of ecological brick prototypes and on the mechanical characterization water absorption, dry density, resistance to mechanical compression; stress/strain behavior, mass behavior/compressive strength. The results showed that the excessive inclusion of recyclable materials in the manufacture of adobes promotes an increase in mechanical resistance, but also increases the absorption of water in a
\end{abstract}


way that does not meet the regulation of the Brazilian standard that provides for the solid brick of soil-cement. The most promising bricks were those made with 55 and $39.5 \%$ recyclable materials for the argisol and oxisol respectively.

Keywords: Adobe; Argisol; Oxisol; PET; EPS; Mechanical compression.

\section{Resumen}

La industria de la construcción utiliza abundantes materias primas no renovables, consume gran cantidad de energía en la extracción de insumos, en la producción de materiales, en el transporte de ambos y genera una cantidad infinita de escombros en la ejecución de las obras. Los ladrillos ecológicos se pueden fabricar utilizando residuos urbanos no tóxicos, incluida la construcción civil. Este trabajo tuvo como objetivo describir el proceso de fabricación y la caracterización estructural de ladrillos ecológicos utilizando como matriz el argisol y el oxisol, y como aglutinante, materiales reciclables de la recogida urbana selectiva (PET y EPS), pasta de cemento tirado por la construcción civil y vinaza de la industria de producción de alcohol. Se realizaron investigaciones sobre el proceso de fabricación de prototipos de ladrillos ecológicos y sobre la caracterización mecánica: absorción de agua, densidad, resistencia a la compresión mecánica, comportamiento tensión/deformación, comportamiento masa/resistencia a la compresión. Los resultados mostraron que la inclusión de materiales reciclables excesivos en la fabricación de adobes promueve un aumento de la resistencia mecánica, pero también aumenta la absorción de agua de una manera que no cumple con la regulación de la norma técnica brasileña para el ladrillo macizo de tierra y cemento. Los ladrillos más prometedores fueron los fabricados con 55 y 39,5\% de materiales reciclables para el argisol y el oxisol respectivamente.

Palabras clave: Adobe; Argisol; Oxisol; PET; EPS; Compresión mecánica.

\section{Introduçãa}

A cultura do desperdício é intrínseca ao setor da construção civil. Esta atividade é considerada um grande gerador de quantidade e diversidade de resíduos que, na maioria das vezes, são dispostos de maneira inadequada (Agopyan \& John, 2016). Segundo dados da Construction \& Demolition Recycling Association, CDRA (2020) no ano de 2019, os Estados Unidos geraram mais de 500 milhões de toneladas de materiais de construção e demolição recuperáveis. Esses materiais incluem concretos, asfaltos, telhas de asfalto, gessos cartonados, madeiras e metais de resíduos de construção e demolição. Embora a atividade da construção civil seja, reconhecidamente, representativa no produto interno bruto (PIB) do país, por outro lado, o setor também possui colaboração significativa na promoção da degradação do meio ambiente (Ajayi, 2017). Além disso, elevadas quantidades de emissão de $\mathrm{CO}_{2}$ é devido ao processamento da matéria-prima da atividade construtiva (Nadoushani \& Akbarnezhad, 2015; Oh, Park, Choi, \& Park, 2016).

Por outro lado, o desenvolvimento sustentável têm sido cada vez mais inserido no âmbito da construção civil (Cordeiro, Masuero, Molin, Souza, \& Paes, 2017). Esse setor, caracterizado por consumir e descartar grande volume de materiais vem buscando ações que visam minimizar os impactos ao meio ambiente por meio do reuso de água nos canteiros, a utilização de materiais ecoeficientes, a redução do consumo de energia e o reaproveitamento de resíduos de construção e demolição (RCD).

Em busca de modelos menos impactantes ao meio ambiente, surge o interesse pelo processo construtivo com o emprego da terra crua, recorrendo à uma arquitetura vernácula. Em sua forma mais pura, materiais de terra crua consistem apenas na mistura e compactação do solo com água, sem maiores transformações industriais (Gallipoli, Bruno, Perlot, \& Mendes, 2017). Ainda, um material de terra, pode conter adição de agregados como areia, cascalho ou fibras, que por sua vez, estão ligados por uma matriz argilosa, fornecendo coesão e resistência mecânica (Fabbri \& Morel, 2020; Harries \& Sharma, 2020).

A tecnologia atual possibilita a incorporação de terra crua em edificações com alguns melhoramentos, no sentido de garantir maior segurança e durabilidade das construções por meio da estabilização do solo com aglomerantes, partículas e/ou nanopartículas, resinas, novos cimentos, cal ou aditivos químicos estabilizantes (Brito \& Paranhos, 2017). O desenvolvimento de materiais construtivos, como tijolos ecológicos se consolida pelo reaproveitamento de resíduos em geral (Klang, Vikman, \& Brattebø, 2003). Além de diferenças dos tijolos tradicionais como na extração de matéria-prima ou recursos não renováveis, os resíduos podem substituir constituintes e ainda oferece produção mais limpa, isenta de queima, pois é substituída por processos alternativos de estabilização (Huarachi, Gonçalves, de Francisco, Canteri, \& Piekarski, 2020; Zhang, Wong, Arulrajah, \& Horpibulsuk, 2018). 
A argamassa para alvenaria colabora para promover a impermeabilização, através de um revestimento que torna as superfícies mais uniformes, mais protegidas das intempéries e com maior aceitação. A geração de resíduos de argamassa para alvenaria é proveniente do desperdício em canteiros de obra. No Brasil, os resíduos da construção civil de classe 'A' (Conselho Nacional do Meio Ambiente, 2002) representam aproximadamente 63\% do total de RCD produzido por ano (Silva Filho, 2005). Entretanto, estes resíduos já possuem utilização consolidada em novos materiais construtivos.

A vinhaça é o efluente gerado especificamente na etapa de destilação para obtenção de etanol. Esse efluente sucroalcooleiro, em altas concentrações, é altamente poluente, devido à combinação de altos teores de matéria orgânica, elevada demanda bioquímica de oxigênio (DBO), pH ácido e a presença de elementos como potássio e enxofre. Porém, possui valor fertilizante dado à sua riqueza em matéria orgânica (75\% dos sólidos totais) e a presença de componentes como nitrogênio, fósforo e potássio (Seixas, Gimenes, \& Fernandes-Machado, 2016). Segundo Corazza; Salles Filho (2014), existem estudos sobre a fabricação de materiais de construção, principalmente tijolos, a partir da vinhaça, tendo avanços significativos quanto à resistência mecânica do material produzido.

Polímeros recicláveis vem sendo utilizados em larga escala e em distintas etapas na construção civil. O polietileno tereftalato (PET), reciclável (garrafa pet), é reaproveitado desde o envase, na produção de elementos decorativos, até como alvenaria de vedação. Quando utilizado em fração micronizada agrega leveza aos concretos, lajes e blocos; e através do processamento, novos produtos como tubos e telhas. Sena; Laursen; Silva (2017) avaliaram o comportamento mecânico de tijolos solo-cimento com adições de PET reciclável em 5, 8 e 11\%. Os resultados mostraram que a incorporação de 11\% de PET, promoveu um aumento de $\sim 163 \%$ comparado aos tijolos de traço padrão. Já o poliestireno expandido (EPS) é aplicado desde a fundação até a cobertura, como em juntas de dilatação, lajes nervuradas e industrializadas, fôrmas para concreto, como isolamento térmico e até como sistema construtivo.

Este trabalho teve como objetivo fabricar e caracterizar mecanicamente tijolos ecológicos do tipo solo-cimento com a utilização de argissolo ou latossolo como matriz, resíduos da construção civil (argamassa reciclável e isopor (EPS)), da coleta seletiva urbana (as garrafas PETs) e como agente hidratante, a vinhaça da indústria energética.

\section{Materiais e Métodos}

O trabalho foi desenvolvido baseado na abordagem quantitativa e experimental (em duas etapas) (Soares, Shitsuka, Parreira, \& Shitsuka, 2018). Na primeira, foi realizada a fabricação dos tijolos ecológicos com diferentes proporções de resíduo de argamassa (RA), PET e EPS. A vinhaça foi utilizada como agente hidratante em substituição a água. Na segunda etapa, foi realizada a caracterização estrutural dos mesmos. Em todo processo, a metodologia quantitativa possibilitou a coleta de dados através de medições em tamanho dos grãos $(\mathrm{mm})$; componentes dos tijolos ecológicos (\%); resistência à compressão (MPa); limite de plasticidade (\%); coeficiente de permeabilidade do solo $(\mathrm{m} / \mathrm{s})$; teor de umidade $(\%)$; densidade $\left(\mathrm{g} / \mathrm{cm}^{3}\right)$; tensão $\left(\mathrm{kgf} / \mathrm{mm}^{2}\right)$ e deformação $(\mathrm{mm})$.

\subsection{Materiais}

Os materiais empregados no processo de fabricação do tijolo ecológico foram os solos classificados como argissolo (A) e latossolo (L). Como aglomerantes, foram utilizados o cimento Portland CP II-Z-32 e a cal hidratada CH III. O RA utilizado apresentava o traço 1:1:5 (cimento: cal: areia), utilizado após sua fragmentação e peneiramento em abertura de malha de \#0,075 $\mathrm{mm}$. As placas de EPS, foram micronizadas e submetidas à separação dos componentes $>1,2 \mathrm{~mm}$. Tanto o RA quanto o EPS foram coletados de resíduos oriundos de uma obra residencial unifamiliar. O PET já micronizado $(<0,6 \mathrm{~mm})$ foi doado por uma empresa de Nova Odessa, estado de São Paulo. A vinhaça utilizada em substituição à água de amassamento foi cedida por uma usina sucroalcooleira de Martinópolis, São Paulo. 


\subsection{Processo de Fabricação dos Tijolos Ecológicos}

Os materiais recicláveis foram adicionados manualmente à matriz de solo (latossolo ou argissolo) e homogeneizados, utilizando como agente hidratante a vinhaça da cana-de-açúcar, para posterior moldagem, prensagem e cura (7 e 28 dias).

Os protótipos de tijolos foram moldados em fôrmas prismáticas nas dimensões 6,6 x 13,8 x 3,0 cm (largura, comprimento e altura) e submetidos a três cargas de prensagem em uma prensa hidráulica (Contenco, modelo UMC) ajustada para 12 toneladas/10 s. Para minimizar o mau cheiro da vinhaça nas peças, foi utilizado a cal com proporção inferior a 10\%.

A definição dos traços, com diferentes dosagens dos materiais recicláveis, partiu de um traço piloto recomendado pelo Boletim Técnico da Associação Brasileira de Cimento Portland, ABCP (2000) que adota a relação de 1:10 (cimento:solo). A quantidade de RA foi determinada a partir de um processo iterativo de confecção de traços onde o cimento era substituído pelo RA até obter tijolos com resistência mecânica compatível à NBR-8492 (ABNT, 2013). Posteriormente foram introduzidos o PET e o EPS que proporcionaram menor utilização de solo e maior leveza aos tijolos, respectivamente.

A Tabela 1 descreve, em porcentagem, os traços, utilizados para o argissolo (A) e o latossolo (L). Os traços 2A e 2L apresentam quantidade intermediária de resíduos, $55,15 \%$ e 39,37\% respectivamente. Os traços 1A e $1 \mathrm{~L}$ foram fabricados com $39,75 \%$ e $28,33 \%$ de resíduos, já os traços 3A e 3L foram produzidos com maior quantidade de resíduos $70,12 \%$ e $54,25 \%$ respectivamente.

Tabela 1 - Composição em massa (\%) dos componentes dos tijolos ecológicos.

\begin{tabular}{|c|c|c|c|c|c|c|}
\hline \multirow[t]{2}{*}{ Componentes } & \multicolumn{3}{|c|}{ Argissolo } & \multicolumn{3}{|c|}{ Latossolo } \\
\hline & 1A & $2 \mathrm{~A}$ & 3A & $1 \mathrm{~L}$ & $2 \mathbf{L}$ & $3 \mathbf{L}$ \\
\hline Solo & 53,79 & 40,04 & 26,68 & 64,68 & 54,72 & 42,10 \\
\hline Óxido de cálcio (cal) & 6,46 & 4,81 & 3,20 & 6,99 & 5,91 & 4,55 \\
\hline Resíduo de argamassa (RA) & 22,45 & 33,42 & 44,54 & 12,13 & 20,52 & 31,58 \\
\hline Polietileno tereftalato (PET) & 4,89 & 7,28 & 9,71 & 2,64 & 4,47 & 6,88 \\
\hline Polietileno expandido (EPS) & 0,13 & 0,19 & 0,26 & 0,14 & 0,12 & 0,18 \\
\hline Vinhaça & 12,28 & 14,26 & 15,61 & 13,42 & 14,26 & 15,61 \\
\hline 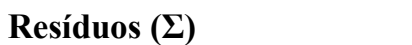 & 39,75 & 55,15 & 70,12 & 28,33 & 39,37 & 54,25 \\
\hline
\end{tabular}

Fonte: Autores.

\subsection{Caracterização Física dos Materiais}

A caracterização física dos solos e dos resíduos (argamassa, PET e EPS) foi realizada pelo ensaio de granulometria (NM-248, 2003) e o ensaio de limites de Atterberg (NBR-6459, 2017; NBR-7180, 2016). Ainda foi realizado o ensaio de permeabilidade (NBR-13292, 1995) com carga constante a fim de verificar se a vinhaça proporcionaria a impermeabilização do solo. A umidade ótima de compactação do solo foi verificada pelo ensaio de compactação pelo método de Proctor normal (NBR$7182,2020)$ que determinou a quantidade ideal de vinhaça a ser utilizada.

\subsection{Caracterização Física dos Tijolos ecológicos}

O ensaio de absorção de água foi realizado de acordo com a (NBR-8492, 2013) de tijolo de solo-cimento. Os ensaios de resistência à compressão mecânica (ABNT NBR-8492, 2013) foram conduzidos em uma prensa hidráulica, Contenco, modelo UMC, com a velocidade constante de $10 \mathrm{~mm} / \mathrm{s}$ e aplicação uniforme da carga. A técnica do ensaio de resistência à compreensão mecânica também permite obter a curva tensão x deformação do material (Callister Jr. \& Rethwisch, 2012). A tensão (MPa) foi calculada pela razão entre a carga aplicada e a área inicial da seção transversal. Já a deformação, pela razão entre: variação do comprimento da amostra em um dado instante e comprimento inicial. 


\section{Resultados e Discussão}

\subsection{Caracterização Física dos Materiais}

\section{Granulometria, Limites de Atterberg e Permeabilidade}

A Tabela 2 sumariza o tamanho dos grãos $(\mathrm{mm})$ dos componentes utilizados na fabricação do tijolo ecológico. Após a fragmentação, o resíduo de argamassa apresentava 100\% do tamanho dos grãos inferior à 0,075 mm, passante na peneira n²00. A cal resultou em $85 \%$ dos grãos inferiores a $0,075 \mathrm{~mm}$. A parte passante do PET e do EPS ficaram retidas nas peneiras $\mathrm{n}^{\circ} 30$ $(0,60 \mathrm{~mm})$ e $\mathrm{n}^{\circ} 16(1,2 \mathrm{~mm})$, respectivamente.

Tabela 2 - Tamanho dos grãos dos componentes do tijolo ecológico.

\begin{tabular}{lr}
\hline \multicolumn{1}{c}{ Componentes } & Tamanho de grãos (mm) \\
\hline Argissolo (A) & $\geq 0,075$ \\
Latossolo (L) & $\geq 0,075$ \\
Resíduo de argamassa (RA) & $\leq 0,075$ \\
Polietileno tereftalato (PET) & $\leq 0,60$ \\
Poliestireno expandido (EPS) & $\leq 1,20$ \\
Óxido de cálcio (cal) & $\leq 0,075$ \\
\hline
\end{tabular}

Fonte: Autores.

O ensaio de limites de Atterberg define, em porcentagem, o limite de liquidez (LL), ou seja, teor de umidade onde em maiores valores o solo adquire o comportamento líquido e, se abaixo o solo se comporta como plástico. Já o limite de plasticidade (LP), representa o teor de umidade ou abaixo deste índice o solo passa do estado plástico para o semissólido, tornando-se quebradiço. O solo do tipo argissolo (A) apresentou LL de 19,29\%; LP de 15,39\% e IP de 3,9\%, em conformidade com as normas NBR-6459 (2017); NBR-7180 (2016). Já a AASTHO (2008) o classifica em A-2, solos granulares se enquadrando em A-2-a ou A-2-4, pedregulhos e areias siltosas ou argilosas. Enquanto o latossolo (L) foi classificado como A-3, solos granulares, areia fina.

Foram obtidas medidas de permeabilidade para amostras de argissolo/água e argissolo/vinhaça. Aos 119 dias, a amostra de argissolo/água permitiu a percolação de $1110 \mathrm{ml}$ e apresentou coeficiente de permeabilidade do solo (k) de $33 \times 10^{-9}$ m/s. A amostra de argissolo/vinhaça não apresentou percolação de água durante todo período de 119 dias.

\section{Ensaio de Proctor Normal}

Para determinar a proporção adequada da vinhaça, não somente em relação ao traço solo-cimento, mas também em relação à presença da cal no traço, realizou-se o ensaio de compactação Proctor normal (NBR-7182, 2020), onde a umidade ótima de compactação é dada pela densidade máxima da amostra, obtida através da relação peso específico máximo por volume. A Tabela 3 apresenta os resultados de compactação Proctor normal (densidade seca $\left(\mathrm{g} / \mathrm{cm}^{3}\right) \mathrm{x}$ teor de umidade $(\%)$ ) para o argissolo e o latossolo na presença de cal, água e vinhaça. 
Tabela 3 - Compactação Proctor normal para amostras de argissolo/cal/água, argissolo/cal/vinhaça, latossolo/cal/água e latossolo/cal/vinhaça.

\begin{tabular}{lcc}
\hline \multicolumn{1}{c}{ Amostras } & Teor de umidade $\mathbf{( \% )}$ & Densidade seca $\left(\mathbf{g} / \mathbf{c m}^{\mathbf{3}}\right)$ \\
\hline Argisolo/cal/água & 14,0 & 1,86 \\
Argissolo/cal/vinhaça & 13,5 & 1,87 \\
Latossolo/cal/água & 14,3 & 1,85 \\
Latossolo/cal/vinhaça & 14,6 & 1,84 \\
\hline
\end{tabular}

Fonte: Autores.

Os resultados obtidos, para as amostras de ambos os solos com água, mostraram que o teor de umidade ideal é de 14,0\% para o argissolo e 14,3\% para o latossolo. Esta diferença de 0,3\% significa que o latossolo requer maior quantidade de água, em relação ao argissolo, para atingir a densidade seca máxima, que foi de $1,86 \mathrm{~g} / \mathrm{cm}^{3}$ para o argissolo e $1,85 \mathrm{~g} / \mathrm{cm}^{3}$ para o latossolo. Nos experimentos com vinhaça, os resultados mostraram que ambos os solos necessitam de maior quantidade de vinhaça para atingir os valores ideais de densidade seca e umidade ótima, quando comparados aos resultados das amostras com água.

Os solos apresentaram comportamentos opostos no que se refere à densidade seca máxima e teor de umidade. As amostras de argissolo apresentaram aumento de $0,5 \%$ na densidade seca máxima e diminuição, também de $0,5 \%$, no teor de umidade em relação as amostras de argissolo com água.

Comparando os resultados das amostras de latossolo/cal/vinhaça com as de latossolo/cal/água foi observado diminuição de $0,5 \%$ na densidade seca e aumento de $0,3 \%$ no teor de umidade ideal. Os resultados apresentados mostraram que a substituição da água pela vinhaça proporcionou o aumento em $0,5 \%$ da densidade seca para as amostras com argissolo e diminuição em $0,5 \%$ da densidade seca nas amostras com latossolo.

\subsection{Caracterização Física dos Materiais}

\section{Compressão Mecânica e Absorção de Água}

Os ensaios de resistência à compressão mecânica dos tijolos ecológicos após cura de 7 dias estão mostrados na Figura 1. Os tijolos fabricados com argissolo apresentaram valores individuais acima de 1,70 MPa, atendendo à norma técnica para solo-cimento (ABNT NBR-8491, 2012). O traço 3A, que possui maior quantidade de resíduos (70,12\%), apresentou em média, o maior resultado de compressão mecânica (3,43 MPa), entretanto, devido à alta quantidade de RA na massa, ocasionou absorção de água acima de 20\%, que não é o permitido pela ABNT NBR-8491 (2012), (Figura 2). 
Figura 1 - Compressão mecânica dos tijolos ecológicos fabricados aos 7 dias de cura.

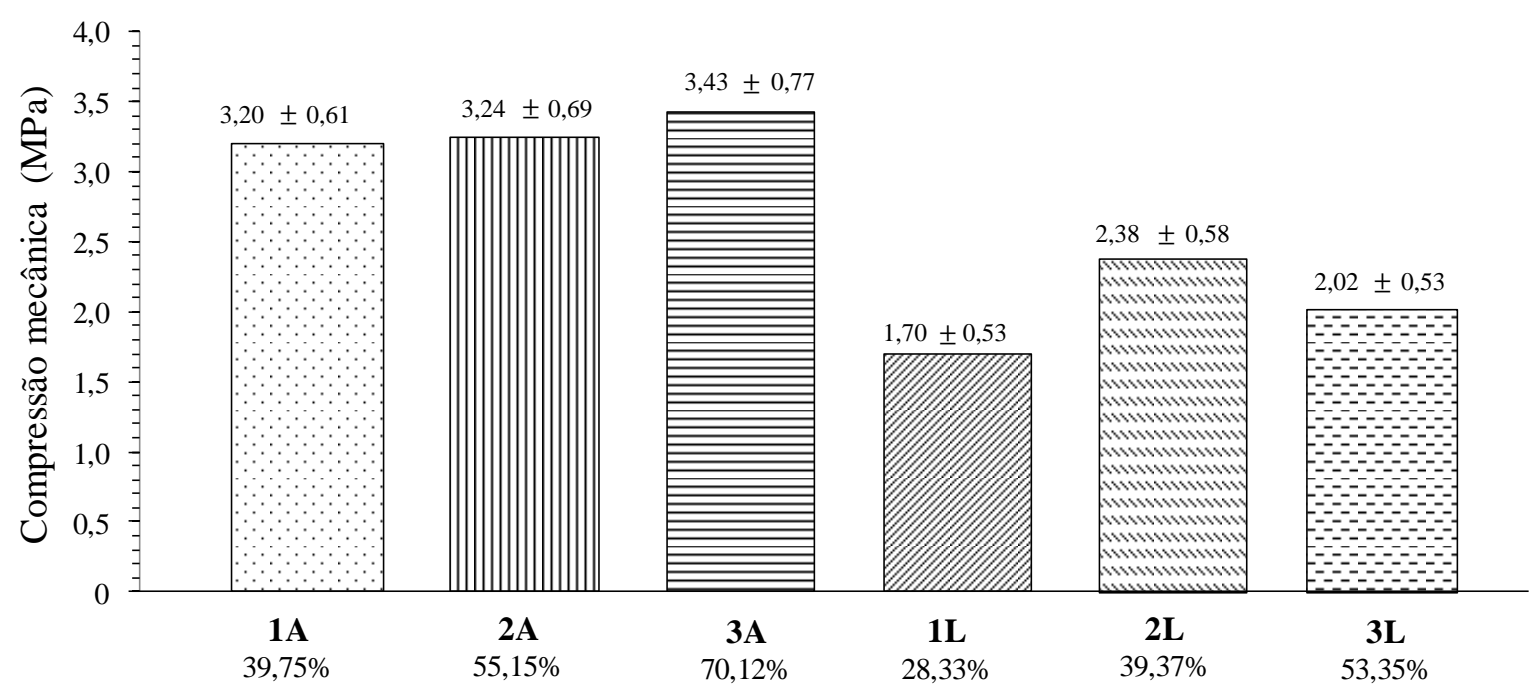

Fonte: Autores.

Figura 2 - Densidade seca $\left(\mathrm{g} / \mathrm{cm}^{3}\right)$ e absorção de água (\%) para tijolos de argissolo (1 A, 2 A e 3 A) e de latossolo (1L, 2L, 3L) com cura de 7 dias.

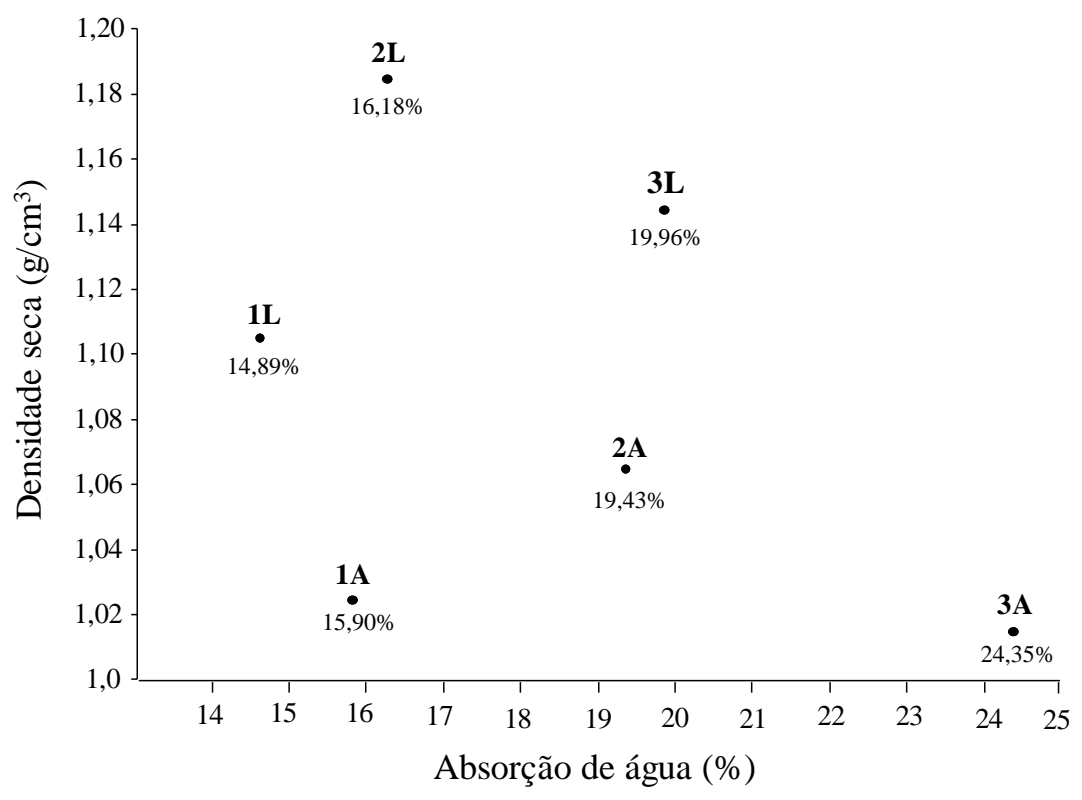

Fonte: Autores.

Os tijolos fabricados com latossolo apresentaram compressão mecânica média de 2,0 MPa e densidade seca superior aos de argissolo (Figura 2). Nota-se que os valores obtidos no ensaio de resistência à compressão são superiores aos apresentados por Rolim (1996), que estudou o comportamento solo-vinhaça para fabricação de tijolos de solo/cimento. Nos solos arenosos obtiveram uma média de 1,92 MPa e nos solos argilosos os valores médios com a utilização da vinhaça ficaram em 1,79 MPa. Valenciano; Freire (2004) ao utilizar cinzas de bagaço de cana-de-açúcar em substituição ao cimento, verificou que a incorporação de $20 \%$ de cinzas de aumentou a resistência à compressão em 60 dias em 23\% comparado aos solos-cimento referência. 
Foram obtidos valores de resistência à compressão para os traços 2A e 2L para corpos de prova com 28 dias de cura. Foi observado o aumento da resistência mecânica para ambos os solos. No intervalo entre 7 e 28 dias a resistência mecânica foi aumentada 22,8\% para o argissolo e 21,7\% para o latossolo.

A densidade seca dos tijolos fabricados com argissolo, foi, em média, 1,02; 1,06 e 1,01 g/ $\mathrm{cm}^{3}$ para os traços $1 \mathrm{~A}, 2 \mathrm{~A}$ e $3 \mathrm{~A}$, respectivamente. Para os tijolos fabricados com latossolo, a densidade seca encontrada foi 1,10;1,18 e 1,14 g/ $\mathrm{cm}^{3}$ para os traços 1L, 2L e 3L. Valenciano \& Freire (2004) ao trabalhar com solo-cimento, observou que nos corpos de prova produzidos com solo arenoso, os valores de massa específica aparente seca foram superiores aos obtidos pelos corpos de prova fabricados com solo argiloso.

Apesar do traço 2A apresentar resistência à compressão de 3,24 MPa, mostrou densidade inferior quando comparado ao traço 2L (2,38 MPa). Isto pode ser explicado devido a maior quantidade de resíduos de baixa densidade, como o PET e EPS, em sua composição e pela presença de silte e argila.

O uso de EPS é eficaz na redução da densidade seca de misturas, entretanto em contato com a água, os vazios presentes em sua própria estrutura são preenchidos, o que modifica sua densidade em condições saturadas (Ali et al., 2020). Lima Jr.; Willrich; Barbosa (2003) também comentam que outras adições minerais, por conta de suas partículas com elevada finura, podem preencher os vazios entre as partículas e aumentar a densidade e a resistência.

\section{Tensão e Deformação dos Tijolos}

A Figura 3 apresenta os resultados do ensaio de tensão $x$ deformação para os lotes dos traços 1A, 2A, 3A, 1L, 2L e 3L após 7 dias de cura. A tensão x deformação destes traços mostrou que o material possui maior comportamento elástico e menor plástico, apresentando o módulo de elasticidade baixo. Em pesquisas, Santos (2009) afirma que a resistência mecânica e o módulo de elasticidade são gradativamente reduzidos na medida em que se aumenta o teor de resíduos.

Os materiais argilosos são em sua grande maioria aluminossilicatos (Walker, 1994). Como são estruturas cristalinas, ao entrar em contato com a água tornam-se tixotrópicos e permitem a moldagem (Barbosa, Amorim, \& Ferreira, 2007). A adição de resíduos como o PET e EPS, não interagem da mesma forma que os agregados naturais, causando uma compactação não homogênea e apresenta variações no regime plástico (traços 1A, 2A e 3A da Figura 3).

Em todos os traços, foi possível observar uma acomodação dos materiais em 0,05 mm de deformação, posteriormente, o comportamento elástico apresentou tendência linear até atingir a tensão elástica máxima, onde ocorreu a deformação plástica por escoamento viscoso, tração máxima e fratura.

No perfil das curvas dos traços $2 \mathrm{~A}, 3 \mathrm{~A}, 2 \mathrm{~L}$ e $3 \mathrm{~L}$ há variações que induzem ao escoamento do material durante a deformação elástica e na deformação plástica pequenas fissuras ou deslizamentos antes da ruptura. Tais irregularidades podem estar relacionadas a dispersão física heterogênea dos materiais recicláveis durante o processo de fabricação dos tijolos, uma vez que a mistura dos materiais foi realizada manualmente. Este comportamento foi observado apenas nos traços com maiores quantidades de materiais recicláveis.

Nos traços de argissolo pode-se observar que com o aumento da incorporação de resíduos houve uma tendência a aumentar as deformações. Este comportamento é desejável, uma vez que a deformabilidade é uma propriedade intrínseca à absorção dos esforços mecânicos e no controle das fissurações causados pelos esforços e movimentações naturais presentes nas estruturas (V. Agopyan, Savastano, John, \& Cincotto, 2005).

Nas amostras 1A e 2A a zona de fluência (zona elástico-plástica) é bem definida e atinge limites de fluência de 0,29 $\mathrm{kgf} / \mathrm{mm}^{2}$ para o traço $1 \mathrm{~A}$ e $0,25 \mathrm{kgf} / \mathrm{mm}^{2}$ para o traço $2 \mathrm{~A}$. 
Figura 3 - Tensão x deformação dos tijolos aos 7 dias de cura.

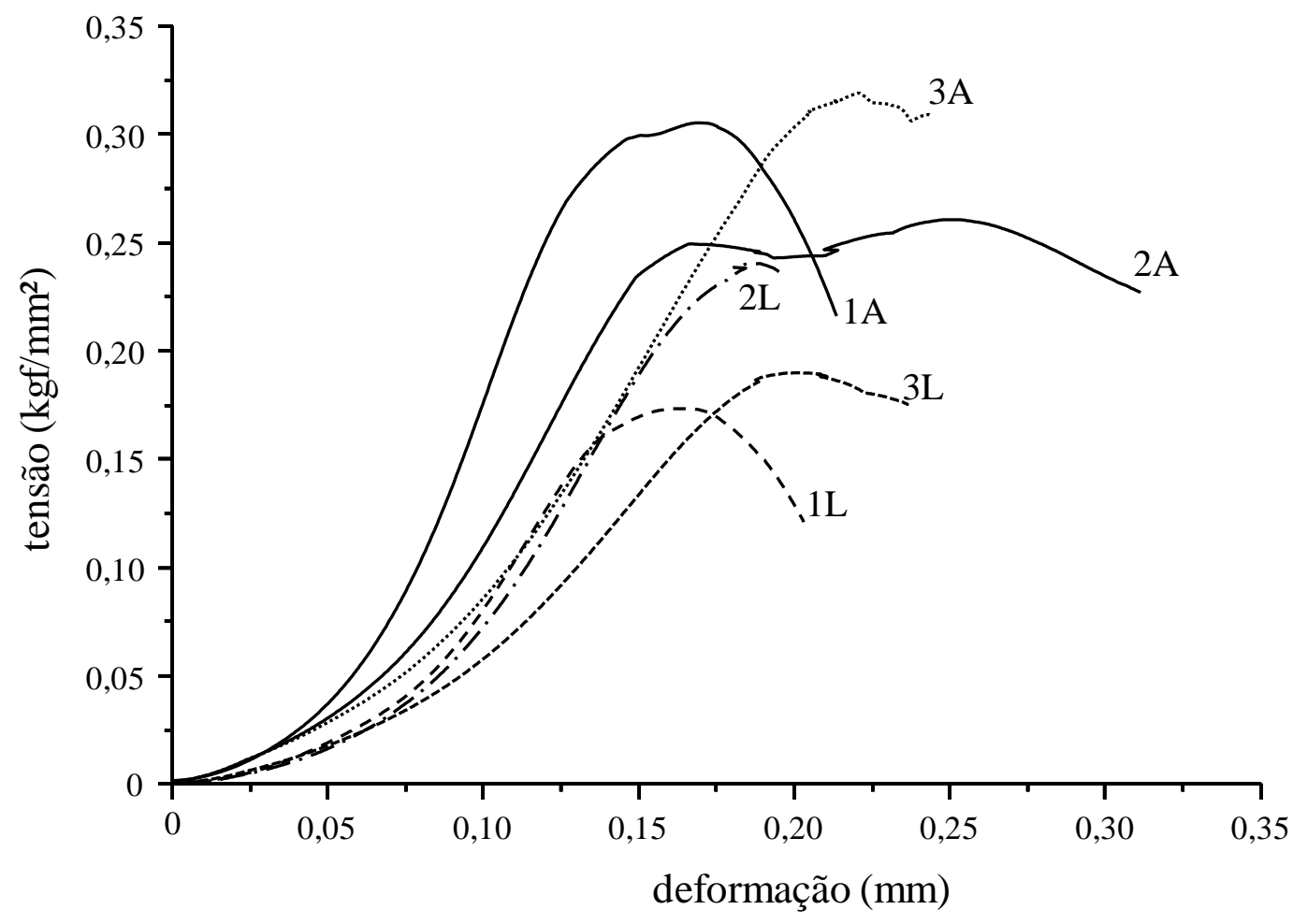

Fonte: Autores.

Complementarmente, realizou-se o ensaio de tensão x deformação com os traços 2A e 2L para amostras com idade de 28 dias. A Tabela 4 mostra os respectivos valores médios do módulo de elasticidade, em MPa, obtidos através do ensaio de compressão dos traços com argissolo e latossolo com tempos de cura de 7 e 28 dias.

Tabela 4 - Módulo de elasticidade (MPa) dos traços com argissolo e latossolo aos 7 e 28 dias de cura.

\begin{tabular}{ccc}
\hline \multirow{2}{*}{ Traços } & \multicolumn{2}{c}{ Dias de cura } \\
\cline { 2 - 3 } & $\mathbf{7}$ & $\mathbf{2 8}$ \\
\hline 1A & 22,76 & - \\
2A & 16,18 & 21,74 \\
3A & 15,44 & - \\
1L & 11,28 & - \\
2L & 13,12 & 19,20 \\
3L & 9,89 & - \\
\hline
\end{tabular}

Fonte: Autores.

As curvas não apresentaram as irregularidades no comportamento elástico visualizadas nas amostras com 7 dias de idade. Além disso, o maior tempo de cura promoveu aumento significativo da resistência mecânica dos tijolos, uma vez que os valores de tensão aumentaram $25 \%$ para o traço $2 \mathrm{~A}$ e $46 \%$ para o traço $2 \mathrm{~L}$ em relação aos traços $2 \mathrm{~A}$ e $2 \mathrm{~L}$ curados por 7 dias. Silva (2005) trabalhou com diferentes resíduos orgânicos para tijolos de solo-cimento e constatou que a incorporação de 3\% serragem de madeira, diminuiu o módulo de elasticidade para 33,45 MPa e, de acordo com Santos (2009) a mistura de solocimento com 3\% de incorporação de resíduos de madeira, apresentou o módulo de elasticidade de 156,6 MPa e com adição de $6,5 \%$ de resíduo, obteve 54,5 $\mathrm{MPa}$. 


\section{Considerações Finais}

Com as informações obtidas no estudo pode-se concluir que o argissolo e latossolo, se mostraram propícios à fabricação de solo-cimento, solo-cimento-vinhaça, bem como à fabricação de tijolos do tipo adobe. Os resíduos de argamassa, PET e EPS, em proporções adequadas (2A e 2L) favorecem o aumento da resistência à compressão mecânica e o tempo de cura aumenta a resistência mecânica em até 46\%. O aumento da incorporação de resíduos ocasiona maior absorção de água.

$\mathrm{O}$ comportamento tensão $\mathrm{x}$ deformação dos tijolos $2 \mathrm{~A}$ e $2 \mathrm{~L}$ mostraram que a elasticidade aumenta à medida que aumenta o tempo de cura. Quanto maior a quantidade de material reciclável, em especial os polímeros, maior é a ductilidade dos tijolos. O tempo de cura aumenta a resistência à compressão, diminui a deformação e aumenta o módulo de elasticidade. Os melhores resultados de resistência à compressão correspondem aos tijolos ecológicos fabricados com argissolo com 55,15\% de resíduos (2A) e latossolo com 39,37\% de resíduos (2L). Ambos são compatíveis com as normas para solo-cimento, ABNT NBR8492 (2013).

Diante de todos estes apontamentos, conclui-se que, há viabilidade na fabricação dos tijolos ecológicos com os diferentes materiais recicláveis, sendo uma opção viável para aplicação de resíduos na produção de materiais construtivos. Recomenda-se que trabalhos futuros realizem a caracterização físico-química da vinhaça de diferentes origens/lotes, avalie a durabilidade dos tijolos a longo prazo e frente intempéries; realize estudos acerca das propriedades térmicas e acústicas.

\section{Agradecimentos}

O presente trabalho foi realizado com apoio da Coordenação de Aperfeiçoamento de Pessoal de Nível Superior - Brasil (CAPES) - Código de Financiamento 001. Os autores agradecem ao PPGMMADRE, à UNOESTE, ao IFSP-PEP, à FCT-UNESP e FCE-UNESP.

\section{Referências}

AASTHO. (2008). Mechanistic-Empirical Pavement Design Guide. Washington, DC: American Association of State Highway and Transportation Officials. ABCP. (2000). Fabricação de tijolos de solo-cimento com a utilização de prensas manuais. Associação Brasileira de Cimento Portland.

ABNT NBR-8492. (2013). Soil-cement brick — Dimensional analysis, compressive strength determination and water absorption — Test method. Associação Brasileira de Normas Técnicas.

ABNT NBR-13292. (1995). Soil - Determination of the coefficient of permeability of granular soil by constant-head - Method of test. Associação Brasileira de Normas Técnicas.

ABNT NBR-6459. (2017). Soil- Liquid limit determination. Associação Brasileira de Normas Técnicas.

ABNT NBR-7180. (2016). Soil — Plasticity limit determination. Associação Brasileira de Normas Técnicas.

ABNT NBR-7182. (2020). Soil - Compaction test. Associação Brasileira de Normas Técnicas.

ABNT NBR-8491. (2012). Soil-cement brick - Requirements. Associação Brasileira de Normas Técnicas.

ABNT NM-248. (2003). Aggregates - Sieve analysis of fine and coarse aggregates. Associação Brasileira de Normas Técnicas.

Agopyan, V., Savastano, H., John, V. M., \& Cincotto, M. A. (2005). Developments on vegetable fibre-cement based materials in São Paulo, Brazil: an overview. Cement and Concrete Composites, 27(5), 527-536. https://doi.org/10.1016/j.cemconcomp.2004.09.004

Agopyan, V., \& John, V. M. (2016). O desafio da sustentabilidade na construção civil (3a ed.),. Blucher.

Ajayi, B. (2017). An assessment of environmental and sustainability provisions in trade agreements. University of Waterloo, Ontario, Canada. https://doi.org/10.1177/0309133309346882

Ali, Y. A. Y., Fahmy, E. H. A., AbouZeid, M. N., Shaheen, Y. B. I., \& Mooty, M. N. A. (2020). Use of expanded polystyrene in developing solid brick masonry units. Construction and Building Materials, 242, 118109. https://doi.org/10.1016/j.conbuildmat.2020.118109

Barbosa, M. I. R., Amorim, L. V., \& Ferreira, H. C. (2007). Compostos poliméricos como aditivos de argilas bentoníticas. Cerâmica, 53(328), 354-360. https://doi.org/10.1590/S0366-69132007000400004

Brito, L. C., \& Paranhos, H. da S. (2017). Estabilização de Solos. Revista Científica Multidisciplinar Núcleo Do Conhecimento, 1(2), $425-438$. 
Callister Jr., W. D., \& Rethwisch, D. G. (2012). Ciência e engenharia de materiais: Uma introdução. (Sergio Murilo S. Soares, Trans.) (V.8). John Wiley \& Soons.

Construction \& Demolition Recycling Association. (2020). Collaborate. Innovate. Advance. https://cdrecycling.org/about/

Conselho Nacional do Meio Ambiente, C. Resolução CONAMA n ${ }^{o}$ 307, de 5 de julho de 2002. Alterada pela Resolução no $348 / 04$ (alterado o inciso IV do art. $3^{\circ}$ ). Estabelece diretrizes, critérios e procedimentos para a gestão dos resíduos da construção civil (2002). Brasil. https://www.mma.gov.br/estruturas/a3p/_arquivos/36_09102008030504.pdf

Corazza, R. I., \& Salles-Filho, S. (2014). Vinasse treatment in Brazil from 1970s to 1990s: a lock-in case study into the ethanol agroindustry. Análise Econômica, 32(62). https://doi.org/10.22456/2176-5456.20928

Cordeiro, L. de N. P., Masuero, A. B., Molin, D. C. C. D., Souza, P. S. L., \& Paes, I. N. L. (2017). Influence of the mixing processes in concrete with aggregates coarse recycled concrete. Ambiente Construído, 17(3), 255-265. https://doi.org/10.1590/s1678-86212017000300174

Fabbri, A., \& Morel, J. C. (2020). Earthen materials and constructions. In Nonconventional and Vernacular Construction Materials (pp. 375-401). Elsevier Ltd. https://doi.org/10.1016/b978-0-08-102704-2.00014-7

Gallipoli, D., Bruno, A. W., Perlot, C., \& Mendes, J. (2017). A geotechnical perspective of raw earth building. Acta Geotechnica, 12(3), 463-478. https://doi.org/10.1007/s11440-016-0521-1

Harries, K. A., \& Sharma, B. (2020). Nonconventional and vernacular construction materials: Characterisation, properties and applications (Vol. 2). Duxford, United Kingdom: Elsevier Ltd. https://books.google.com.br/books?id=geu-DwAAQ BAJ\&pg=PA399\&lpg= PA399\&dq=Lei,+ X.,+et+al.,+2014.+A+thermochemo-electro-mechanical+framework+of+unsaturated+expansive $\quad$ clays.+Comput.+Geotech.+ $\quad 62,+175 \mathrm{e} 192 \&$ sour $\quad$ ce $=$ bl\&ots $\quad=A$ 0FOPMEXAM\&sig=ACfU3U0esjYSTsm1UvGwyts4NS

Huarachi, D. A. R., Gonçalves, G., de Francisco, A. C., Canteri, M. H. G., \& Piekarski, C. M. (2020). Life cycle assessment of traditional and alternative bricks: A review. Environmental Impact Assessment Review, 80, 106335. https://doi.org/10.1016/j.eiar.2019.106335

Klang, A., Vikman, P.-Å., \& Brattebø, H. (2003). Sustainable management of demolition waste-an integrated model for the evaluation of environmental, economic and social aspects. Resources, Conservation and Recycling, 38(4), 317-334. https://doi.org/10.1016/S0921-3449(02)00167-2

Lima Jr., H. C., Willrich, F. L., \& Barbosa, N. P. (2003). Structural behavior of load bearing brick walls of soil-cement with the addition of ground ceramic waste. Revista Brasileira de Engenharia Agrícola e Ambiental, 7(3), 552-558.

Nadoushani, Z. S. M., \& Akbarnezhad, A. (2015). Effects of structural system on the life cycle carbon footprint of buildings. Energy and Buildings, 102, 337346. https://doi.org/10.1016/j.enbuild.2015.05.044

Oh, B. K., Park, J. S., Choi, S. W., \& Park, H. S. (2016). Design model for analysis of relationships among CO2 emissions, co st, and structural parameters in green building construction with composite columns. Energy and Buildings, 118, 301-315. https://doi.org/10.1016/j.enbuild.2016.03.015

Rolim, M. M. (1996). Avaliaçao físico-mecânica do material solo-vinhaça concentrada e sua utilizaçao para fins de fabricaçao de tijolos. Universidade Estadual de Campinas, Campinas, São Paulo, Brasil.

Santos, M. P. dos. (2009). Fabricação de solo-cimento com adição de resíduos de madeira provenientes da construção civil. Universidade Federal de Minas Gerais, Belo Horizonte, MG, Brasil.

Seixas, F. L., Gimenes, M. L., \& Fernandes-Machado, N. R. C. (2016). Treatment of vinasse by adsorption on carbon from sugar cane bagasse. Química Nova, $X Y(200), 1-8$. https://doi.org/10.5935/0100-4042.20160013

Sena, R. J. De, Laursen, A., \& Silva, J. S. da. (2017). Mechanical evaluation of solid soil-cement brick containing PET residue. Veredas MPCT, 10(1), 69-83.

Silva, S. R. da. (2005). Tijolos de solo cimento reforçado com serragem de madeira. Universidade Federal de Minas Gerais;

Silva Filho, A. F. e. (2005). Gestão dos resíduos sólidos das contruções prediais na cidade do Natal-RN. Universidade Federal do Rio Grande do Norte, Natal, Brasil. Retrieved from http://repositorio.ufrn.br:8080/jspui/handle/123456789/14974

Soares, A. P., Shitsuka, D. M., Parreira, F. J., \& Shitsuka, R. (2018). Metodologia da pesquisa científica. UFSM.

Valenciano, M. D. C. M., \& Freire, W. J. (2004). Características físicas e mecânicas de misturas de solo, cimento e cinzas de bagaço de cana-de-açúcar. Eng. Agríc, 24(3), 484-492. https://doi.org/10.1002/0471478768.ch13

Walker, P. J. (1994). Properties of stabilized soil blocks. In 5th International Seminar on Structural Mansory for Developing Countries. Brasil.

Zhang, Z., Wong, Y. C., Arulrajah, A., \& Horpibulsuk, S. (2018). A review of studies on bricks using alternative materials and approaches. Construction and Building Materials, 188, 1101-1118. https://doi.org/10.1016/j.conbuildmat.2018.08.152 